fluence of the Madeira climate. In these two concluding cases, for instance, the benefit has been as striking though the state of the lung is less satisfactory.

CAsE vrir. E. S., aged 31, widow; dressmaker, had had a cough for three years and night-sweats. In August 1865, on the right ride there were cavernous gurgling; crepitation and prolonged expiration. On the left side, expiration was harsh and prolonged. Cod-liver oil, quinine, and iron were preseribed; and gallic acid to check hæmoptysis. The patient lived near Paris. The physical signs are now, dulness on the right side, with cracked-pot sound; dry cavernous respiration over the upper half of the chest. The left side is normal. She is now able to work.

CA8E Ix. G. J., aged 25, a barman, in September 1865, had a cough, profuse hæmoptysis; night.sweats, and emaciation. The right side was dall; with cracked-pot sound, humid crepitation, and rhonchus to the base. On the left side were rhonchi and sibilus. Cod-liver oil, iron, and zinc pills, were prescribed.

February 1867. The right side is flat and dull, and there are bronchial breathing and bronchophony. The patient has returned to his occupation.

\section{CASE OF}

\section{PARALYSIS OF THE DIAPHRAGM: WITH REMARKS.}

BY P. VICTOR BAZIRE, M.D.LOND. \& PARIS, Assistant-Physieian to the National Hospital for the Paral ysed and Epileptic.

Ir is, I believe, the general impression among medical men, that paralysis of the diaphragm is an immediately fatal affection, and cannot be seen clinically. Between seven and eight years ago; how. ever, I happened to see at the Charité Hospital in Paris, a well-marked example of this affection in an hysterical young woman, who was under Dr. Briquet's care, and $I$ then learnt how to recognise and treat it. Dr. Duchenne, (of Boulogne) who has done so much for neuropathology, a few years before that time, had been the first to show that the diaphragm, although the chief inspiratory muscle, might act imperfectly or not at all, might be more or less paralysed in fact, without instant death or even fatel consequences following, so long as the patient remained tree from any pulmonary complication.

The mistaken opinion which is current in the pro. fession, has, I imagine, arisen from the circumstance that immediate death follows an injury to the spinal cord, sufficiently high in the neck as to be above the origin of the third and fourth cervical nerves, from which the phrenic nerve is mainly derived. The important fact has been overlooked that, in sach cases, all the muscles concerned in the great function of respiration are simultaneously deprived of power; that the intercostal muscles are paralysed together with the auxiliary muscles of respiration, and that hence arise the asphyxia and the rapid death. When the diaphragm alone is affected, tranquil respiration may be carried on seemingly with little trouble; and it is not until the patient taxes his breathing powers to a greater degree than usual by fast walking, running, or exertion of any kind; that he becomes aware that something is wrong with his breathing on account of the oppression which he feels at his chest, and is alarmed at finding that his roice either leaves him entirely, or is reduced to a mere whisper. In the following case, which has been for some time under my care at the National Hospital for the Paralysed and Epileptic, the chief symptoms of paralysis of the diaphragm, as indicated by Duchenne, will be seen to be fwell-marked; and, although the patient has been conscious of a difficulty of breathing, brought on by the least exertion of an unustial kind, for fifteen months, and unquestionably traceable to defective action of the diaphragm, she is still alive and enjoying on the whole pretty good health.

CASE I. Sarah B., aged 41, married, a tall, thin, bony woman, of pale sallow complexion has always enjoyed good hoalth, and has never been laid up for ary illness. The only member of her family who has saffered from a nervous complaint is her eldest son, aged 14, a remarkedly well-developed boy, who looks more like a strong lad of 20 , and who has been for some time past under my care for epilepsy; which now recurs at very long intervals only. She is of a nervous, excitable temperament, and confesses to be easily moved, and to be subject to depression of spirits, but denies having ever had any hysterical seizures, of any fits of crying and sobbing.

Her present complaint dates from November 1865 . It began very gradually, with a sense of discomfort about the epigastrium (not exactly a pain, bat a sensation of weight) and a difficilty of breathing; she could not fetch her breath, she says, and kept gaping so as to take in more air inside her chest. These sensations came on towards evening, and were most marked when she had had a hard day's work to do, a good deal of washing and ironing to get through. She was living poorly at the time, and, as she had to work hard, she felt herself becoming weaker. The difficulty in breathing was accompanied by a symptom which considerably alarmed her, namely, loss of voice. Frequently, towards evening, she would lose her voice completely, and be compelled to speak in a mere whisper. If she abstained, throughout the day, however, from work requiring a good deal of muscular exertion, and confined herself to mere sewing, for instance, she was free from dyspncod, and could speak in a louder voice. She was always better in the morning; and, if she had gone to bed' voiceless; would wake up after a good night's rest perfectly able to speak. She had no cough at the time, and no pain about the chest to account for the difficulty of breathing; and she felt no soreness; no abnormial sensation in the throat, to which she might refer her occasional and intermittent aphonia.

When I first saw her, the peculiar character of her voice attracted $m y$ attention at once. She spoke like a person who has just been running and is out of breath, and stops between the words to draw in air; with this difference, however, that, instead of her voice improving as she went on talking, it became weaker and weaker, occasionally squeaky, till at last it left her completely, and she could not for a while proceed even in a whisper.

I was struck with the peculiarities of this aphonia, especially as the patient pointed of herself to the epigastrium as the seat of an unpleasant sensation, not to the larynx; while, on examination, no redness of the fauces, no swelling of the torsils, was found to exist.

On exposing her chest, her breathing, when she was perfectly quiet, seemed to be regular and normal, and was not visibly accelerated; but on application of the hand, it was found to be in reality quicker than in health, for the respiratory movements were thirty-two in a minute, and the breathing was acertained to be costal. There was some lateral dilatation of the chest; but the increase in capacity was chiefly obtained by the upward movement of the thoracic parietes: No appreciable change took place in the opigastrium during vary tranquil breathing, 
but, as soon as the patient was asked to inspire deeply, her epigastrium was observed to sink invards instead of becoming prominent as it should normally do. The hypochondria became depressed also, and were separated from the base of the chest by a deep groove. There was no perceptible lateral expansion of the base of the chest. During expiration, the parts which had sunken in during inspiration, bulged outwards. On applying the hand over the epigastrium during a forcible inspiration, no abrupt rising of that part could be felt, as should normally be done, from the descent of the contracting diaphragm. The patient could not go on breathing fast, or imitate panting respiration; any attempt at this caused her considerable distress, although the sterno-mastoid, scaleni, and clavicular portion of the trapezii could be seen to contract with energy and to pull the chest-walls upwards.

Although respiration could be but imperfect, through the absence of diaphragmatic action, a sufficient amount of air was apparently taken in for the purposes of ordinary tranquil breathing, for there was no lividity of the face or lips pointing to deficient oxygenation of the blood. On auscultation the lungs were found to be everywhere permeable to the air, and percussion elicited a fair amount of resonance over the front and back of the chest. There ware no enlarged glands at the root of the neck which might cause the aphonia by involving the recurrent laryngeal nerves, nor were there any signs of aneurismal dilatation of the arteries at the base of the neck. The heart was regular in its action, though with a somewhat feeble impulse, its sounds were not very loud, but they were normal, both at base and aper, and no suspicion of disease could attach to the organ; the pulse was regular, 65 in the minute.

On galranising the phrenic nerves in the neck, the diaphragm was made to contract, and a distinct though feeble stroke was felt during inspiration on applying the hand over the epigastrium, while the part was seen to bulge slightly.

The patient has now been submitted to this treat. ment (galvanisation of the phrenic nerves in the neok) for the last air weeks, and has been taking in tarnally, three times a day, five grains of citrate of iron, and ten minims of tincture of nur romica, to one ounce of water, and two drachms of cod-liver oil. Her general health is considerably better, but the condition of her diaphragm has not materially im. proved, and, from the feebleness with which it re. sponds to galvanic excitation, I suspect that the mascle is structurally disorganised.

A review of the chief symptoms exhibited by this patient will show that they are those which Dr. Duchenne (of Boulogne) has proved by clinical observations, and by experimental demonstration, to characterise paralysis of the diaphragm. (Seo his Traité de l'Electrisation localisée, p. 718 : and his recent work, Physiologie des Mouvements, p. 620 and following.) First and foremost, we find perversion of the normal movements of the epigastrium and hypochondria during respiration. This perversion is not very marked during tranquil breathing, although it is perfectly appreciable even then; but it is when the patient is asked to breathe deeply and rapidly that it becomes peculiarly striking. Instead of the bulging of those parts which obtains during normal inspiration, there is sinking in, depression of the epigastrium, and production of a deep furrow or groove along the base of the chest. In expiration, on the contrary, the epigastrium bulges when it should normally sink inwards. In a case reported by Duchenne, the patient complained, whenever she took a very deep breath, of a sensation as if her ab. dominal viscera rose into her chest; but in the pre. sent instance no such sensation was ever felt. Even when the patient was perfectly quiet, and the eye detected nothing abnormal with the respiratory movements, an acceleration of the respiration could be detected by applying the hand over the chost. On connting the number of respirations, it was found to be nearly double the average in health, and yet there was no corresponding increase in the number of pulsations at the wrist, and auscultation and percussion of the chest detected no abnormal condition of the intrathoracic organs. Intermittent aphonia had been the first symptom to alarm the patient, and it was all the more striking that it was not accompanied by any sign of disease about the larynx or throat; there was no soreness of those parts, no sensation of pricking, no cough, no ab. normal symptom whatever referable to them. After her admission, laryngoscopic examination detected nothing abnormal about the glottis; and the rocal cords could be seen to approximate normally, when she was asked to exclaim, ha ! ha! The nurse of the ward and the other patients confirmed the original statement made by the patient, that she could speak with a clear, distinct voice when she got up in the morning, but, as the day wore on, her voice failed gradually in power, and towards evening it either left her entirely, or was reduced to a mere whisper, with an occasional squeak. Any unusual exertion, fast walking, going up a staircase, brought on breathlessness, aphonia, and a sensation of weight and discomfort about the base of the chest.

Paralysis of the diaphragm has been noted in hysteria, in general paralysis from plumbism, in progressive muscular atrophy, and in some inflammatory conditions of the thorax and abdomen (empyema and peritonitis.)

Many cases of hysterical aphonia are, I suspect, instances of this affection, and will probably be recognised as such when the diagnostic signs of diaphragmatic paresis come to be better known. The subject of the first case of this affection which came under my notice was an hysterical woman, and Duchenne gives the particulars of a very marked instance of the kind in his work on Localised Electrisation. The same author reports a case of general paralysis from plumbism, in which the symptoms of paralysis of the diaphragm existed at one time, and ultimately disappeared as the patient recovered his health. In the remarkable work of Tanquerel des Planches, on the diseases caused by lead, two cases of aphonia are recorded, as occurring in individuals suffering from the effects of chronic lead. poisoning; and A. Portal (in Cours d'Anatomie Médicale, Paris, 1804, vol. iv, p. 361) mentions two other instances of the kind. The aphonia was ascribed to paralysis of the laryngeal muscles, as the most probable cause, but may have been due, as in the analogous case reported by Duchenne, to a paralytic condition of the diaphragm.

An observer of established reputation, Dr. Aran, of Paris, noted the signs of paralysis of the diaphragm in a case of metro-peritonitis, in which a post mortem examination subsequently showed that the inflammation had spread upwards, and involved the diaphragm.

In an essay, published in tho Dublin Journal of Medical Science, by Dr. Stokes, in 1836, ("Researches on Laennec's vesicular emphysema," etc.) that eminent physician expressed the opinion that the disphragm was occasionally paralysed in empyema, and suggested that the presence of this condition might serve to diagnose purulent effusion within tho chest from mere hydrothorax. Duchenne, however, has shown that, although the eminent Dublin physician 
was correct in stating that the diaphragm $\mathbf{w a s}$ occasionally paralysed in empyema, the signs which he mentions as indicating this complication are quite the reverse of what obtains in such cases. For Dr. Stokes regards permanent bulging of the epigastriun as a sign of paralysis of the diaphragm, whereas, as Duchenno was the first to show oxperimentally, as well as clinically, perversion of the normal movements of the epigastrium and hypochondria, during respiration, is tho only sign which can be depended upon. In a case of pleuritic effusion in which t'is perversion was noted by Duchenne and others, the liquid effused was found after death to be puralent, while the diaphragm was seen to be inflamed. The plan recommended by Duchenne for recognising this morbid condition consists in laying the palm of the hand flat on the hypochondriumone on each side-and watching its movements as the patient takes a deep breath. The hand on the side of the empyema will be seen to sink, and that on the sound side to rise during inspiration, and the latter will feel a distinct impulse as the contracting half of the diaphragm descends.

In that curious affection, progressive muscular atrophy, the diaphragm, like other striated muscles, may become involved, but only at a very advanced stage of the complaint. The muscles of the extremities are the first to atrophy (and, in the immense majority of cases, the disease first begins in the hand), and disorganisation of the muscles of the trunk will precede, for a considerable period, the implication of the diaphragm. From the distress of breathing, which is apt to come on at an advanced stage of this complaint, one is not to argue atrophy of the diaphragm. For, if the dyspnose bo not accompanied by occasionally complete aphonia, (not mere feebleness of the roice) and by the characteristic depression of the epigastrium during inspiration, it will be found, on eramination, to be due to wasting of the intercostal muscles.

In a paper, published in a recent number of the Lancet, (January 12th, 1867) Mr. Callender has called attention to cases of fatty degeneration of the diaphragm. He relates six instances in which this condition, which had not been suspected during life, was revealed by a post mortem examination, and he suggests that death may have been due to it through failure of the respiration; but in all these cases, there was coexistent fatty degeneration of the heart, a condition perfectly sufficient to account for the sudden fatal termination, and for the symptoms which immediately preceded dissolution.

The diagnosis of paralysis of the diaphragm may be readily effected by means of the two prominent symptoms indicated by Duchenne, epigastric depression during inspiration, and aphonia of a peculiar character.

As to the cause of the paralysis, it may be made out by a consideration of the other symptoms of disease, if any, exhibited by the patient, and by the effects noted on galvanising the phrenic nerves. In cases of hysteria, the previous history of the patient will tell of hysterical fits, after one of which the characteristic symptoms of paralysis of the dia. phragm will probably have shown themselves. In progressive muscular atrophy, the striking manifestations of that complaint in other regions of the body will at once attract attention; while in empyema and peritonitis there will be evident indications of those affections. If the disease be due to impregnation of the system with lead, the rell. known signs of this condition will sufficiently point to that cause; the blue line on the gums, and the characteristic dropping of the wrist from paralysis of the extensor muscles of the hand, and loss of the electro-muscular contractility of the paralysed mus. cles.

If all the above causes can be excluded, fatty de. generation of the muscle may be suspected, and this suspicion be considered as a certainty, if signs of fatty degeneration be present in other organs, such as the heart, liver, or cornea.

The effects obtained by galvanising the phrenic nerres will at once indicate whether the paralysis of the diaphragm is due to a structural alteration, or is merely functional. In the latter case, the muscle will contract powerfully when galvanic irritation is transmitted to it through its nerve; in the former, it will respond feebly or not at all to the excitation, according to the degree of structural alteration.

Prognosis, in paralysis of the diaphragm, is of course subordinate to the cause which induced the paralysis. It is, as a rule, favourable in hysteria. In general paralysis from plumbism, Duchenne has known the complaint get well as the lead was eliuninated from the system; and he has even seen it disappear, in a case of progressive muscular atrophy, under the influence of a treatment by galvanisation of the phrenic nerves. Although not an immediately fatal affection, as is generally believed, paralysis of the diaphragm is at all times a very serious condition, as it may cause the patient's death on the supervention of the least bronchitic attack or other thoracic complication. - The respiration is already so imperfect and incomplete, that any additional interference with the proper oxygenation of the blood rapidly induces a condition of asphyria, of which death is the only termination. An individual, therefore, who presents the symptoms of paralysis of this important muscle, should be carefully protected against the risks of any thoracic affection; and means should be taken to try and restore contractile power in the palsied muscle. The best treatment for obtaining this is the one recoinmended by Dachenne; namely, galvanisation of the phrenic nerres. In one class of cases, however, electricity should not, I believe, be had recourse to; namely, in cases of suspected fatty degeneration of the muscle, if there be at the same time a feeble action of the heart, and other signs indicating probable fatty decay of that organ. All emotional excitement should be carefally avoided in such cases; and the certain amount of shock which always follows the use of electricity, especially in weak nervous individuals, might prove fatal to a person with a feebly acting fatty heart. The phrenic nerve may be galranised in the neck, just above the clavicle, close to the outer border of the sterno-cleido-mastoid, where it lies immediately beneath the skin and platysma myoides in front of the anterior scalenus. The skin should be well stretched and depressed with the fingers, and the conductor pressed hard down, so as to make the current pass deeply inwards. Duchenne recommends the use of insulated conductors with conical metallic extremities, covered with chamois leather, which is well wetted previous to being used; and they are un. questionably more manageable than sponges. The current should be weak to begnn with, and be very gradually and carefully increased; and the circuit should be interrupted every two or three seconds, so as to imitate as much as possible natural respiration. It is not an easy thing to galvanise the phrenic. In some cases, the platysma myoides is so developed that it contracts powerfully, and impedes the inward passage of the current; in others, the carrent is disseminated-dispersed, as it were-and acts on all the muscles of the neck, and on the nerres which go to form the brachial plexus. Sometimes, again, the phrenic, soon after its origin, inclines considerably to the median line, and gets under cover of the 
aterno-mastoid. As a rule, the conductors have to be moved along the side of the neck before they are successfully placed over the course of the nerve. When the phrenics are fairly galvanised, the epigas. trium and hyprchondria are seen to bulge; while, on applying the hand over the epigastrium, a distinct stroke may be felt, imparted to it by the descent of the contracting diaphragm. The patient makes at the same time a characteristic gasping inspiration.

\section{PUERPERAL CONVULSIONS BEFORE} DELIVERY : DEATH : REMARKS.

By C. J. E V A N S, Esq., Northampton.

Mrs. C., a stout, florid, bealthy-looking woman, 24 years of age, and within a month of her first confine. ment, being as well as usual in the afternoon of March 11th, 1867, with the exception of a little pain in the stomach, which she attributed to having eaten some beef, was suddenly seized at twelve o'clock at night with convulsions. Mr. Cotton kindly saw her for me in my absence, and ordered leeches to be applied to the temples, a sinapism to the back of the neck, cold to the head, and a dose of calomel to be taken immediately.

I visited her at two o'clock in the morning (March 12th), and found her quite insensible, the convulsions occurring about every half-hour; the respiration was stertorous; the pupils were rather contracted than not; the remains of the calomel to be seen on her tongue. I directed the same treatment to be continued.

9 A.x. The convulsions had continued at intervals during the night, and the stupor was now profound. Only three of the leeches had taken hold. The pulse being as high as 120, full and bounding, I at once bled her to nearly two pints. Its force and rapidity were thereby considerably lessened, and she became more conscious than she had previously been, opening her eyes when loudly spoken to.

Two drops of croton-oil were placed on the tongue, and a saline draught, with a powder composed of a grain of calomel, was ordered to be taken every two hours. The hair was cut close to the head, and cold directed to be constantly applied. No urine having been passed, $I$ introduced the catheter and drew off about half a pint, which was dark and muddy (but not smoky), and was so loaded with albumen as to bocome perfectly solid on applying the usual tests. The os uteri was at this time just capable of admitting the point of the finger, which detected a headpresentation. The os showed a slight tendency to dilate.

No improvement in the symptoms occurred during the morning.

2 P.x. Mr. Cotton saw the patient with me in consultation. The convulsions were still occurring at intervals. The os uteri was now dilated sufficiently to admit the ends of three tingers; and we agreed that delivery should not be delayed a moment longer. I accordingly gradually introduced my hand, turned the child, and delivered; the child (a female) being atill-born. The placenta came away almost immediately, without hæmorrhage. The woman was quite unconscious during the whole time of delivery, and remained in that state afterwards, for the convulsions occurred as before, and she died a short time subsequently.

This case suggests one or two points worthy of consideration, especially as regards the treatment.

On the morning after the commencement of the attack, finding that the previous treatment had been of no avail, and the pulse being full, rapid, and bounding; considering also the stout, plethoric condition of the patient-I did not hesitate to abstract blood freely from the arm, not, however, without having first ascertained that I could not at that time have the benefit of Mr. Cotton's opinion and advice; and the immediate effect of the bleeding, though only temporary, appeared to my mind at the time to justify me in the step I had taken.

In the Medical Times and Gazelte for February 23rd, 1867, p. 191, is a very interesting communication on this subject by Dr. Arthur S. Donkin, in which he details three cases, proving, in his opinion, the value of blood-letting (venesection), but which seem hardly to bear out bis faith in this particular mode of treatment. Two out of the three cases related ended fatally, and the third recovered. In the farourable case, the convulsions were preceded for some time by premonitory symptoms; whereas, in the fatal cases, the convulsions themselves were the first symptoms; and the value of blood-letting may have been great or little, according as the disease manifested itself gradually or suddenly.

In a case under the care of Dr. Graily Hewitt, related at page 353 of the Britrs Medical JourNal for March 30th, 1867, a totally opposite plan of treatment was adopted-riz., stimulation; Dr. Hewitt being of opinion that the convulsions were due to the loss of blood itself which had occurred before delivery. Bleeding in this case was, of course, out of the question; and the result of the case would appear to militate against such practice. On the other hand, Churchill, Denman, Blundell, and others, recommend bleeding, and that largely.

It is only right to state that Mr. Cotton, who kindly assisted me in the case, afterwards informed me that he denounces venesection, and places his reliance on local bleeding.

M. Trousseau, in his Clinical Lectures, translated and edited by Dr. Bazire, states that he does " not include in the treatment of eclampsia general or local bleeding," on the principle that the cerebral congestion is the effect, and not the cause, of the convulsions. On the contrary, he recommends antispasmodics, and especially the inhalation of chloroform. The convulsions do not necessarily in every case occur with congestion of the brain, but mas be accompanied with, if not caused by, effusion of serum into the ventricles; and in such cases, bleeding, either general or local, is neither necessary nor advisable. The case under the care of Dr. Hewitt would appear to have been of this nature.

There seems to be considerable difference of opinion as to the propriety of delivering the patient artificially, and also as to the mode by which delivery shall be effected; but the forceps would appear to be most in rogue. It is certain, however, that they could not safely be used so early in the case as the process of turning; which latter is also attended with less danger to the mother under the circumstances.

It appears to be pretty generally allowed that albuminous urine coexists with this form of convalsions (it was present to an extreme degree in the above case); and further, that it may exist to a greater or less extent for some time previously during pregnancy. Such being the case, it is somewhat remarkable that the general health does not more frequently suffer beforehand; for I suppose it will be admitted that the symptoms of convulsions do not present themselves, in the greater majority of cases, until at or near the setting in of parturition. At that time, however, the uterus commences to descend into the pelvis, and so its pressure on the renal system of ressels would be less than it had been previonsly. 